\title{
Optimisation for Surface Mount Placement Machines
}

\author{
${ }^{1}$ Masri Ayob , ${ }^{2}$ Peter Cowling, And ${ }^{3}$ Graham Kendall. \\ ${ }^{1,3}$ Automated Scheduling, Optimisation and Planning (ASAP) Research Group, \\ CSIT, University of Nottingham, Nottingham NG8 1BB, UK \\ Phone: +44-115-846-6525 Fax: +44-115-951-4254 E-mail: mxa|gxk@cs.nott.ac.uk \\ ${ }^{2}$ MOSAIC Research Group, Computing Department, Bradford University, Bradford BD71DP, UK. \\ E-mail: Peter.Cowling@scm.brad.ac.uk
}

\begin{abstract}
Optimisation of feeder setup and component placement sequence are very important to the efficiency of surface mount placement machines. Much works have been conducted to solve this problem. However, the technological characteristics of the placement machine influences the nature of the planning problems to be solved and the formulation of the associated models. As a result, little consensus exists as to what a suitable model should be for a given machine characteristics, and the formulations proposed by different authors tend to be difficult to compare. Hence, this paper will survey the relation between models, assembly machine technologies and heuristic methods.
\end{abstract}

Keywords: Modelling, Optimisation, Electronic Assembly, Printed Circuit Board Assembly, SMT.

\section{Introduction}

When hundreds of electronic components of different shapes and sizes have to be placed at specific locations on a printed circuit board (PCB), finding an optimal robot travelling route is complicated and time consuming [1]. This problem is an NP-Hard problem and most practical instances are difficult to solve to optimality in a reasonable time [2]. In practice, a heuristic solution is highly desirable [1]. Heuristic algorithms can generate good solutions efficiently at a reasonable computational cost [3]. Khoo and Loh [4], for example, have developed a prototype genetic algorithm (GA) to enhance a planning system for the placement of surface mount devices (SMDs) on a Fuji FCP-IV. Wang et al.[3] argue that their GA performs as well as a human expert in optimising the feeder slot assignment problem for the Fuji QP-122. Crama et al. [5] agree that the technological characteristics of the equipment influences the nature of some of the planning problems to be solved and the formulation of the associated models. Little consensus exists as to what a suitable model should be for a given set of machine characteristics, and the formulations proposed by different authors tend to be difficult to compare. Hence, this paper will discuss the relation between models, assembly machine technologies and heuristic methods.

Optimisation in PCB assembly involves a list of subproblems to be addressed, such as an assignment of PCB types to product families and to machine groups, allocation of components to machines and location of components in feeder slots and component placement sequencing [5]. In this work we focus on feeder setup and component placement sequencing for various type of placement machine for surface mount technology (SMT). More general reviews on PCB assembly problems can be found in $[5,6]$.

\section{Placement Machines}

Placement machines are sometimes called "chip shooters" [3]. There are many types of SMT placement machines available, such as sequential pick-and-place, rotary disk turret, concurrent pick-and-place, etc. [4,7]. Various types of SMT placement machines have different characteristics and restrictions [3]. Thus, the PCB production scheduling process is highly influenced by the type of placement machine being used [8]. Industrial robotic placement machines have already been classified by mechanical structure such as cartesian/gantry, cylindrical, spherical, single compliance robot for assembling (SCARA), articulated and parallel [9]. However, the mechanical structure classifications do not greatly influence the nature of optimisation problems since most of the placement machines used in PCB assembly industry are cartesian/gantry robots. Thus, in this work we propose five categories of machines based on their specification and operational methods. Previously, the placement machines have been classified into two categories these being concurrent and sequential by McGinnis et al. [6], or fixed pick-and-place point (FPP) and dynamic pick-and-place point (DPP) by Wang et al. [10]. However, these two categories are too general, hence it tends to be difficult to formulate the optimisation problems based on these categories.

Generally, each placement machine has a feeder carrier (or feeder magazine), PCB table, head, nozzle (or gripper) and a tool magazine. The feeder carrier, PCB table and head can be either fixed or moveable depending on the specification of the machine. In some cases, the feeder carrier is divided into separate feeder banks, each consisting feeder slots [3]. A typical feeder carrier consists of either several tape reels or vibratory ski slope feeders or both [11]. The feeder reels or vibratory ski slope feeders are positioned in the feeder slots according to the arrangement given by feeder setup. The nozzle grasps the component from the feeder and then mounts it on the PCB [12]. The placement arm, that is equipped with head(s), are responsible for picking and placing components. Each head may have more than one nozzle and each machine may have more than one head. There are various types of placement heads, such as a rotating turret head, or a positioning arm head [3]. The PCB table(s) are required to position the $\mathrm{PCB}(\mathrm{s})$ during placement operation. The table(s) could be stationary, a conveyor system, or an X-Y motion table. Different sizes of SMD require different sizes of nozzle to pick-and-place them. Hence, a tool magazine is required to provide the exact nozzle size.

\subsection{Dual Delivery Placement Machine}

This machine consists of the PCB table which can move in both $\mathrm{X}$ and $\mathrm{Y}$ directions and should be aligned under the 
head to perform the placement operation; the placement arms and two component delivery carriers are only able to move in the X-direction $[11,13]$. The pick-and-place heads are mounted at the two ends of a fixed length arm, which can move between two fixed positions in the Y-direction only. Each pick-and-place operation alternates between two sides, i.e. while one head is performing the pick operations, the other one is placing components on the board [13]. For this machine, all movements of the PCB table and feeder carrier are frozen during the pick-andplace operations. Therefore, the maximum time taken by the arm, PCB table and feeder carrier movements will determine the cycle time. The Dynapert MPS 500 [13], the Panaset MCF that is equipped with 10-nozzle gang pickup [14], the Fuji NP-132 which contains dual turret placement heads with 16 nozzles on each head and the Siemens Siplace 80S-20 [15] are all examples of dual delivery placement machine.

\subsection{Multi-Station Placement Machine}

This machine has more than one placement module (or station) each one being mechanically identical and able to assemble electronic parts concurrently. The stations are connected by a conveyor system to transfer boards among the stations. Each station receives all the necessary pickand-place coordinate data for one machine cycle (the interval between two conveyor steps), and completes the cycle's placement sequence autonomously and concurrently with the other stations. After all stations have finished, the conveyor is moved, and the placement procedure continues. The Fuji QP-122 [3, 16] that has 16 stations with each station consisting of fixed multi-feeder unit and a single-nozzle placement head is an example of the multi-station placement machines.

\subsection{Turret Style Placement Machine}

This machine uses a placement mechanism mounted on a rotating turret (drum or carousel), with multiple placement heads, that rotate from a fixed pickup location to the placement location [17]. Each pick-and-place operation starts by retrieving a component at the grip station, while the placement station simultaneously mounts a component at a pre-specified location on the PCB $[2,18]$. Then, the feeder rack moves to get the next appropriate feeder in position, and the $\mathrm{PCB}$ table simultaneously moves to position the next location under the place station. Some of the common turret style placement machines include the Fuji FCP-IV [19, 20] that has 12-nozzles mounted on a rotary head, the Fuji CP4, CP4-2, and CP4-3, which have 12 mounting heads, the Fuji CP6 which has 20 placement heads [21] and the CM82 equipped with 18 heads [22].

\subsection{Multi-Head Placement Machine}

In this machine, the tour of the heads begins by picking up a few components from the feeder simultaneously. Then, the head and the arm travel in the $\mathrm{X}$ and $\mathrm{Y}$ direction simultaneously and the head positions itself on top of the point where the component will be mounted, and then the head moves down (Z-direction) and mounts the component on the board then returns to the original position and repeats these steps for the next locations on the board that have to be mounted on the same tour. The PCB table also moves in $\mathrm{X}$ and $\mathrm{Y}$ directions, concurrent with the movements of head and arm. After completing a tour, the head returns to the feeder location to begin another tour, unless nozzle changes are required. The heads of this machine can be similar to the heads of turret type machine. The difference is that it is located on top of the arm [12]. A head is used to grasp a few components from the feeder locations and mount them on the PCB (e.g. the Quad 400 series [12]).

\subsection{Sequential Pick-and-Place Machine}

According to Kumar and Li [23], the typical machines of this type have placement head mounted at the end of an arm. The arm can move in the X-direction only, simultaneously with the head movement in Y-direction. The nozzle on the head can move in Z-direction to perform pick-and- place operations. The placement arm starts by moving to the tool magazine to equip itself with the proper nozzle. Next, it moves to pick a particular component from the feeder location, and then place the component at the appropriate location on the board. If the following component is of the same type, the arm moves directly to feeder slot to perform the subsequent pick-and-place operation. Otherwise, the arm goes to tool magazine [24, 23]. The Quad IIIC is an example of this machine type [24].

\section{Models And Heuristics}

Four basic PCB component placement rules are usually adopted [25]: (a) sequence the component placement for minimum routing time, (b) arrange feeder reels so as to minimise component pickup time, (c) place identical SMDs in one pass, and (d) sequence placement according to component size. Some works have addressed the problems of feeder setup and placement sequence independently by making assumptions about the rest of the problem, and some prefer to solve both problems as an integrated solution [2]. The question of where (i.e. in which slots) the feeder reels should be attached in each placement machine is referred to as feeder setup [15], feeder rack assignment problem [18], component-feeder arrangement [4], reel positioning problem [13, 22], feeder assignment [24, 26], feeder allocation [12], or magazine assignment [10]. In this paper we use the term feeder setup refer this problem.

\subsection{Models and Heuristics for Dual delivery}

An Adaptive Simulated Annealing algorithm has successfully been applied by Tirpak et al. [15] to solve the feeder, nozzle and placement optimisation problems for the Fuji NP-132. Each iteration of the algorithm requires two main steps: generate a candidate solution and determine if the solution is accepted. Each candidate solution includes a nozzle setup, a feeder setup, and a placement sequence for the two heads. Cheapest insertion and nearest neighbor path construction heuristics are used to construct a placement sequence. A constraint satisfaction swapping heuristic is applied to generate feeder and nozzle setups. The results tested in a Motorola factory show a 3\%-12\% improvement over the original assembly times. Since feeder movement is a critical issue for improving the performance of this machine, Grotzinger [7] and Ahmadi 
et al. $[11,13]$ both addressed this problem in their work. They identified a hierarchical framework consisting of three optimisation problems; the component allocation and partitioning, the placement sequence, and feeder setup.

\subsection{Models and Heuristics for Turret Type}

Since the PCB table moves simultaneously and independently in $\mathrm{X}$ and $\mathrm{Y}$-directions, the chebychev distance can be used to determine PCB table movement time [27]. The turret rotation time is dictated by the component with the slowest turret rotation rate loaded in the turret since larger and heavier components are more difficult to hold in place by the suction nozzle and must move slower [2]. Francis et al. [27] have modeled the feeder setup problem for this machine as a quadratic assignment problem, since the feeders are assigned to slots on the feeder carriage and the cost of the assignment is impacted by the location of other feeders.

Klomp et al. [18], view a feeder (and its corresponding cluster i.e. set of locations served by a single feeder) as a node in a complete graph. Computational result show that the gap between the solution found and the lowerbound is relatively small (about 20\% in the three machine case), which implies that much of PCB table and feeder rack movements fall within the turret rotation time.

Kumar and Luo [19] view the placement sequencing problem on a Fuji FCP-IV, as a "generalised Traveling Salesman Problem (TSP)" where not only the overall travel time depends on the travel sequence (as in standard TSP), but even the distances between any pair of nodes is sequence dependent (whereas in the standard TSP such distances are constant regardless of travel sequence). Since feeder carriage movement is considerably slower than the PCB table movement and turret rotation, and furthermore, these three operations occur concurrently, the whole process is dependent up on the feeder carriage movement. Then they show that an optimal tour for the distance matrix, $d_{i j}$ provides a desired optimal placement sequence (for a given slot assignment) such that it visits all components of the same part number consecutively. If switching components is required, then the feeder carriage should be moved to the adjacent feeder slots in order to obtain the optimal solution. They show consistent improvement of over $25 \%$ in overall assembly time compared to the solution generated by the chip placement machine optimiser at Lexmark, Inc. For some cases, the rotation of the turret, which takes fixed time, determines the travel time, and thus implies that their optimisation algorithm will be more efficient on machines with faster turret rotation or with smaller rotation angles.

Khoo and Loh [4] employed a genetic algorithm (GA) to generate the component placement sequence and feeder setup by formulating it as a multi-objective optimisation problem constrained. The prototype system has demonstrated the ability to generate a component placement sequence and feeder setup slightly better than Leu et al.[28].

More recently, Ellis et al. [2] have developed heuristics for feeder setup and component placement sequencing by using a constructive heuristic that groups together the components with similar PCB table speed and turret rotation speed. The constructive heuristic uses the surrogate function which can provide a method to approximate penalties for feeder carriage movements, changes in turret rotation speed and changes in PCB table speed. After the initial feeder setup and placement sequence have been constructed, the two-opt heuristic is applied to search for placement time improvement. Results indicate that the solutions are close to lower bound and the computational time required to generate the initial solutions is minimal (less than 3 minutes). However, the computational time to generate the improved solution is high, and increases as the problem size increases. For instance, the initial solution computation time is 2 seconds while improvement solution requires 1586 seconds for the smallest PCB. Larger PCBs requires 164 seconds to generate initial solution and 43200 seconds to compute the improved solution.

\subsection{Models and Heuristics for Multi-Station}

Csaszar et al. [16] conducted research to optimise the multi-station machine, which has a single head and a single nozzle per station. The system was designed to emulate human experts. They divided the allocation problem into two sub-problems, that is the assigning of components to the stations, and the arrangement of components within station to achieve maximum throughput by minimising the head movements. The expert system is split into four phases: simulator pre-processing, placement, refining and conversion phases. The results show that the expert systems uses an average of $16.14 \%$ fewer feeder slot than the vendor's software and throughput improve by $13.47 \%$ to $15.95 \%$. They prove that by using the cost function of the number of placements together with pick-and-place time they gain better results than using just pick-and-place time.

\subsection{Models and Heuristics for Multi-Headed}

Since the arm and head can move simultaneously in both the $\mathrm{X}$ and $\mathrm{Y}$ axis, Altinkemer et al. [12] used the chebychev distance and calculated the distance as the maximum of the movements in the $\mathrm{X}$ and $\mathrm{Y}$ direction. They consider two cases; when the feeder locator moves and when the feeder locator does not move. When the feeder locator moves, the feeder of the component type that will be processed next can move towards the tool magazine while the head is mounting another component type, so the distance between the feeder locations and the points on the PCB can be measured from a fixed point next to the tool magazine. The simultaneous movement enables each component type to have the same origin and destination point, and thus allow the formulation to be an independent capacitated vehicle routing Problem. Since the distance between a point on the PCB and feeder is not dependent on where the component is located among feeders, the feeder setup problem does not have to be integrated with the pick and place sequencing decisions. For the case where the feeder locator does not move, they formulate the problems as a combination of assignmentlike and vehicle-like problems. They first solve a vehicle routing problem (VRP) for each component type at every possible feeder location, and then use this feasible solution as the cost of assigning the component type to the particular feeder location. They argue that their integrated 
algorithm provides a feasible solution with an error gap less than or equal to the maximum error gap of the VRP costs.

Lee et al. [29] developed heuristics which are based on dynamic programming and the nearest neighbor TSP to solve the optimisation of multi-head surface mount machine. They choose a hierarchy method to find the solution by starting with the construction of reel-groups, then the assignment of reel-groups and finally sequencing of pick-and-place movements. Since nozzle changes are time-consuming and the number of nozzle changes is proportional to the number of nozzles to be used, they choose a nozzle for each reel in such away that the total number of nozzles is minimised. Then, they assign the reels to heads in such away that each head has about the same workload. Since nozzle changes are the most timeconsuming operation, they decided to first determine the order of nozzle changes before determining the sequence of pick-place movements. The simulation results indicate that their method achieves an average saving of $18 \%$ in PCB assembly time over the heuristic algorithm supplied by Yamaha.

Burke et al. [8], have introduced a three phase heuristic to deal with the assembly of multiple printed circuit board types with different batch sizes on a single machine without set-ups between board types. Experimental result shows that their approaches are very promising.

\subsection{Models and Heuristics for Sequential Pick-and- Place}

Kumar and Li [23] model the optimisation of feeder setup and component placement sequence for sequential pickand-place machine as an instance of a linear integer programming problem. They solve the problem by determining an assignment of pickup slots and a component assembly sequence for each individual nozzle. Heuristics such as nearest neighbour, nearest insertion, furthest insertion, and random generation are used to construct an initial assembly sequence, and the other heuristics such as 2-opt, 3-opt, and Or-opt are used to improve upon the initially generated assembly sequence. Simulation results show a consistent assembly time saving of $25 \%$ over the current approach used in the factory.

Ball and Magazine [30] formulated the placement sequence problem as a type of directed postman problem. They show that balance and connect heuristic can be applied to this problem. Ahmadi and Mamer [31] have modelled the problem of sequencing the part types for placement and the problem of scheduling the movement between points on the PCB as a collection of interdependent travelling salesman problems. The computational results shown that the approximation of the problem by a sequence of TSPs was able to produce significant increases in throughput.

$\mathrm{Fu}$ and $\mathrm{Su}$ [32], and Hop and Tabucanon [26] strongly believe that robotic travel routing should be based on relative coordinates to obtain a better solution because the robotics, board and magazine are simultaneously moved at different speeds during assembly. Their dynamic pick and place (DPP) model has been introduced by $\mathrm{Su}$ et al. [33]. In the DPP model, the robot moves vertically along the Yaxis (in the optimal condition), while the PCB table and feeder rack move horizontally along the X-axis, and the pickup and placement point are dynamically allocated. The optimal condition occurs when the robot travels only in the $\mathrm{Y}$ direction, and no movement in the $\mathrm{X}$ direction is observed [10]. Wang et al [10] have applied off-line heuristics to improve the placement sequence and feeder setup to take advantage of the DPP model. They sequence the placement first by considering the adjacent placement to be as close as possible (minimum travel in $\mathrm{X}$ direction) to achieve minimum total travel while placing each component in its proper location. Next, they organise feeder slots to let all adjacent slots have the highest exchange frequency pickups of the same component type. The exchange frequency is defined as an index that counts the exchange frequency between two different component types for succeeding pickups. They claim that the approach provides a rapid solution to even very large problems, and demonstrate the on-line implementation to ensure the feasibility of the approach. Nevertheless, Wang's approach was still based on a fixed coordinate system using the TSP method to obtain robotic travel routing [34]. To resolve such a dynamic combinatorial problem, $\mathrm{Fu}$ and $\mathrm{Su}$ [32] have applied Genetic Algorithms (GA), Simulated Annealing (SA) and Tabu Search (TS). In the GA approach, a possible solution is represented by chromosome, and each gene in the chromosome represents the insertion point and component. The fitness function is defined as the cycle time of the robotic assembly. In the SA and TS algorithms, the possible solution is represented by vector. The cycle time of the robotic assembly is defined by the energy function in SA or the objective function in TS. These approaches can simultaneously arrange the insertion sequence and assign the magazine slots and yield a better performance compared to the conventional approach (fix pick-and-place model). Computational experiments indicate that the GA's performance is the worst among these approaches in a larger number of insertion points and/or component types. A GA requires more computational time for the larger size of tested cases for a near optimal solution to be obtained. The performance of TS is better than the performance of the other two approaches in smaller number of problem sizes. SA is a robust technique that has better performance in a large number of problem sizes if the computational time is limited. Implementation results also demonstrate that the proposed approach is superior to that of Wang's approach.

More recently, Hop and Tabucanon, proposed two new heuristic algorithms that are multiple criteria approaches [26] and extended DPP (EDPP) model [35], to improve Wang's approach. The multiple criteria approach uses the trade-off between two strategies: minimise the PCB table travelling distance where the strategy is 'assemble by area', and minimise the feeder rack travelling distance where the strategy is 'assemble by component type'. The principle is to select components to assemble which have the same component type as close to each other as much as possible. The algorithm starts with an initial solution from Wang's [10]. Then a new feeder setup is constructed based on criteria assembly by component type. Next, the assembly sequence is generated based on the principle of assemble by area. These two steps are iterative, and will be 
terminated by a stopping criteria. Computational results indicate that the total reduction time ranges between $4 \%$ for lower density board, to $28 \%$ for higher density board compared to Wang's approach. The main difference between DPP and EDPP is that the EDPP considers not only the robot arm motion to be the problem's objective, but also the feeder motion and PCB table motion [26]. The result suggests that the EDPP model is superior to the DPP model.

\section{Conclusions}

Our survey found that based on the specification and operational methods, placement machines may be arranged into five categories: dual-delivery, multi-station, turret style, multi-head and sequential pick-and-place. By classifying placement machines into these categories based on their common characteristics and operational methods, we have shown the relation between models, assembly machine technologies and heuristic methods. Most researchers highlight that the crucial moves which are subject to optimisation are the feeder carrier movements [7, 19, 31]. Currently, there are few works that consider the DPP model in pick-and-place sequencing. Interesting results have been reported by applying the DPP model to the sequential placement machine and it is claimed that the DPP model is superior to the fix-pick-and-place (FPP) model. Therefore, the application of this model to other type of placement machines in future may give good solutions.

\section{References}

[1] Su, C. -T and Fu, H. -P., A Simulated Annealing heuristic for robotics assembly using the dynamic pick-and-place model, Prod. Planning and Control, 1998, 9(8), 795-802.

[2] Ellis, K.P, Vittes, F.J. and Kobza, J.E., Optimizing the Performance of a Surface Mount Placement Machine, IEEE Transactions on Electronic Packaging Manufacturing, 24(3), July 2001, pp. 160-170.

[3] Wang, W., Nelson, P.C. and Tirpak, T.M., Optimization of high-speed multistation SMT placement machines using evolutionary algorithms, IEEE Transactions on Electronics Packaging Manufacturing, 22(2), April 1999, pp. 137 -146.

[4] Khoo, L. P. and Loh, K. M., A Genetic Algorithms enhanced planning system for surface mount pcb assembly, Int. J. of Adv. Manufacturing Technology, 16(4), 2000, pp.289-296.

[5] Crama, Y., Klundert, J.V.D. and Spieksma, F.C.R., Production planning problems in printed circuit board assembly, working paper GEMME 9925, Université de Liège, 1999.

[6 ] McGinnis, L.F., J.C. Ammons, M. Carlyle, L. Cranmer, G.W. Depuy, K.P. Ellis, C.A. Tovey and H. $\mathrm{Xu}$, Automated process planning for printed circuit card assembly, IIE Transactions: Scheduling \& Logistics, 24, 1992, 18-30.

[7] Grotzinger, S., Feeder assignment models for concurrent placement machines, IIE Transactions, 24, 1992, 31-46.

[8] Burke, E. K., Cowling, P. and Keuthen, R., the printed circuit board assembly problem: heuristic approaches for multi-headed placement machinery, Proc. of the IC-AI2001, Las Vegas, CSREA Press (2001), pp. 1456-1462.

[9] Samsung electronics: robots, url: http://www.samsungelectronics.com/factory_automat ion/robots/, Samsung Electronics Co., Ltd., 2001.

[10] Wang, C., Ho, L.-S. and Cannon, D.J., Heuristics for assembly sequencing and relative magazine assignment for robotic assembly, Comp. and Ind. Eng., 34, 1998, pp.423-431.

[11] Ahmadi, J., S. Grotzinger and D. Johnson, Component allocation and partitioning for a dual delivery placement machine, Operations Research ,36, 1988, pp. 176-191.

[12] Altinkemer, K., B. Kazaz, M. Koksalan and H. Moskowitz, Optimization of printed circuit board manufacturing: Integrated modeling and algorithms, European Journal of Operational Research, 124, 2000, pp. 409-421.

[13] Ahmadi, J., Ahmadi, R., Matsuo, H. and Tirupati, D., Component fixture positioning for printed circuit board assembly with concurrent operations, Operations Research 43, 1995, pp. 444-457.

[14] Panasonic Electronic Component Assembly System, 2001.

[15] Tirpak, T.M., Nelson, P.C. and Aswani, A.J., Optimization of revolver head SMT machines using adaptive simulated annealing (ASA), Electronics Manufacturing Tech. Sympoium, 2000, Twenty-Sixth IEEE/CPMT Int., pp.214-220.

[16] Csaszar, P., Nelson, P. C., Rajbhandari, R. R. and Tirpak, T. M., Optimization of automated high-speed modular placement machines using knowledge-based systems, IEEE Transactions on System, Man, and Cybernetics-Part C: Applications and Reviews, 30(4), Nov. 2000, pp. 408-417.

[17] Burke, E.K., Cowling, P.I. And Keuthen, R., New models and heuristics for component placement in printed circuit board assembly, Proc. Of the 1999 IEEE on ICIIS99, pp.133-140.

[18] Klomp, C., Klundert, J.J.V.D., Spieksma, F.C.R.and Voogt, S. The feeder rack assignment problem in pcb assembly: a case study, Int. J. of Prod.Economics 64, 2000, pp.399-407.

[19] Kumar.R. and Luo. Z., Optimizing the operation sequence of a chip placement machine using TSP model, IEEE Transactions on Components, Packaging, and Manufacturing-Part B: Advanced Packaging (Submitted).

[20] Crama, Y., Flippo, O.E., Klundert, J.J.V.D. and Spieksma, F.C.R., The assembly of printed circuit boards: A case with multiple machines and multiple board types, European Journal of Operational Research, 98, 1997, pp.457-472 .

[21] Fuji Product catalog, 2001.

[22] Ohno, K., Jin, Z. and Elmaghraby, S.E., An optimal assembly mode of multi-type printed circuit boards, Computers and Industrial Engineering, 36, 1999, pp.451-471.

[23] Kumar, R. and Li, H., Integer Programming Approach to Printed Circuit Board Assembly Time 
Optimization, IEEE Transactions on Components, Packaging and Manufacturing Technology, Nov. 1995. 18(4), pp 720-727.

[24] Loh, T.S, Bukkapatnam, S.T.S., Medeiros, D. and Kwon, H., A Genetic Algorithm for sequential part assignment for PCB assembly, Computer and Ind. Eng. 40, 2001, pp. 293-307.

[25] Wong, H. and Leu, M.C., Adaptive genetic algorithm for optimal printed circuit board assembly planning, Annals CIRP, 42(1), 1993, pp. 17-20.

[26] Hop, N.V and Tabucanon, M.T., Extended dynamic point specification approach to sequencing robot moves for PCB assembly, Int. J. Prod. Research,39(8), 2001, pp.1671- 1687.

[27] Francis, R.L., McGinnis, L.F. and White, J.A., Facility Layout and Location: An Analytical Approach, Englewood Cliffs, NJ:Prentice-Hall, 1992.

[28] Leu, M.C., Wong, H. and Ji, Z., Planning of component placement/insertion sequence and feeder setup in PCB assembly using genetic algorithm, J. Electronic Packaging, Transactions ASME, 115, 1993, pp. 424-432.

[29] Lee, S. H., Lee, B. H. and Park, T.H., A hierarchical method to improve the productivity of a multi-head surface mounting machine, Proc. Of the 1999 IEEE on Robotics and Automation, Michigan 1999, pp. 2110-2115.

[30] Ball, M.O. and M.J. Magazine, Sequencing of insertions in printed circuit board assembly, Operations Research 36, 1988, pp.192-201.

[31] Ahmadi, R.H. and Mamer, J.W., Routing heuristics for automated pick and place machines, European Journal of Operational Research 117, 1999, pp.533552.

[32] $\mathrm{Fu}, \mathrm{H} .-\mathrm{P}$. and $\mathrm{Su}, \mathrm{C}$. -T., A comparison of search techniques for minimizing assembly time in Printed Wiring Assembly, J. of Production Economics, 63, 2000, pp 83-98.

[33] Su, Y.S., Wang, C., Egbelu, P.J. and cannon, D.J., Dynamic points specification approach to sequencing robot moves for pcb assembly, Int. J. Computer Integrated Manufacturing, 8(6), 1995, pp. 448-456.

[34] Su, C., L. Ho and H. Fu,A novel tabu search approach to find the best placement sequence and magazine assignment in dynamic robotics assembly, Integrated Manufacturing Systems 9, 1998, 366-376.

[35] Hop, N.V and Tabucanon, M.T., Multiple criteria approach for solving feeder assignment and assembly sequence problem in PCB assembly, Production Planning and Control 12(8), Dec 2001, 736-744 . 